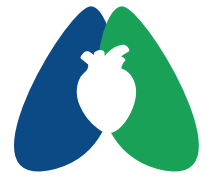

ASSOBRAFIR

C I Ê N C I A

\title{
Capacidade funcional de usuários de narguilé: uma revisão integrativa
}

\author{
Functional capacity of water pipe smokers: an integrative review
}

Micaelly Schell ${ }^{1}$ (i); Karoliny dos Santos Isoppo ${ }^{1 *}$ (1)

\begin{abstract}
Resumo
Introdução: O consumo de narguilé vem crescendo e está sendo associado à diversas repercussões negativas na saúde dos indivíduos, inclusive sobre a capacidade funcional. Objetivo: Realizar uma revisão integrativa da literatura acerca do comportamento da capacidade funcional em usuários de narguilé. Métodos: A busca dos estudos foi realizada nas bases: Biblioteca Digital Brasileira de Teses e Dissertações, Biblioteca Virtual em Saúde, LILACS, PubMed, SciELO e Scopus. Resultados: Foram incluídos quatro artigos, os quais utilizaram para avaliação da capacidade funcional o teste de caminhada de seis minutos e o teste de esforço cardiopulmonar. Estes estudos demonstraram que usuários de narguilé apresentam uma redução na capacidade de exercício e no consumo de oxigênio. Além disso, verificaram-se elevações significativas da frequência cardíaca e da sensação de dispneia durante os testes. Conclusão: Usuários de narguilé apresentam redução significativa da capacidade de exercício. Este achado reforça a necessidade de ações de conscientização sobre os malefícios causados por esta modalidade de tabagismo.
\end{abstract}

Palavras-chave: Cachimbos de Água; Tolerância ao Exercício; Fumantes; Tabaco.

\begin{abstract}
Background: The water pipe consumption has been growing and it is being associated with several negative repercussions on the health of individuals, including the functional capacity. Aim: To carry out an integrative literature review about the functional capacity in water pipe users. Methods: The search for the studies was carried out from the databases: Brazilian Digital Library of Theses and Dissertations, Virtual Health Library, LILACS, PubMed, SciELO and Scopus. Results: Four articles were included, in which the six-minute walk test as well as the cardiopulmonary exercise test were used to assess functional capacity. These studies have shown that water pipe users have a reduction in exercise capacity and oxygen consumption. In addition, there were significant increases in heart rate and dyspnea during the tests. Conclusion: Water pipe users have significantly reduced exercise capacity. This finding reinforces the need for actions to raise awareness about the harm caused by water pipes.
\end{abstract}

Keywords: Smoking Water Pipes; Exercise Tolerance; Smokers; Tobacco.

${ }^{1}$ Departamento de Fisioterapia, Universidade do Sul de Santa Catarina (UNISUL), Palhoça, SC, Brasil

\section{Como citar: Schell M, Isoppo KS. Capacidade funcional de usuários de narguilé: uma revisão integrativa. ASSOBRAFIR Ciênc. 2022;13:e44266. https:// doi.org/10.47066/21779333.AC.2020.0037}

Submissão em: Agosto 02, 2021 Aceito em: Novembro 16, 2021

Estudo realizado em: Universidade do Sul de Santa Catarina, Palhoça, SC, Brasil. Aprovação ética: Não se aplica.

*Autor correspondente:

Karoliny dos Santos Isoppo.

E-mail: fisio.karoliny@gmail.com

Este é um artigo publicado em acesso aberto (Open Access) e distribuído sob a licença Creative Commons Attribution NonComercial ShareAlike License, que permite uso, distribuição e reprodução em qualquer meio, sem restriç̃̃es desde que sem fins comerciais e que o trabalho original seja corretamente citado e de forma que não indique endosso ao trabalho feito. Adicionalmente, qualquer trabalho derivado deverá ser publicado sob a mesma licença. 


\section{INTRODUÇÃO}

O narguilé é um dispositivo para fumar, originário da Índia, no qual uma mistura de tabaco é aquecida e a fumaça passa por um filtro de água antes de ser aspirada. O tabaco geralmente é saborizado e sua queima é acompanhada pela queima de carvão'. A queima deste é usada como fonte de calor, e a fumaça contém produtos tóxicos emitidos tanto pelo carvão quanto pelo produto de tabaco².

O uso do narguilé vem crescendo globalmente, sendo muito popular entre jovens, entretanto o conhecimento da população em relação aos efeitos que o narguilé exerce sobre a saúde é pouco disseminado. Ainda que pareça menos prejudicial que o cigarro, o narguilé tem sido associado a vários desfechos prejudiciais à saúde, como doenças cardiovasculares, respiratórias, e até mesmo câncer ${ }^{3-5}$.

Sabe-se que o tabagismo pode aumentar a fadiga durante a realização de exercícios, reduzir a capacidade aeróbica máxima e diminuir o desempenho durante a realização de testes funcionais, mesmo em indivíduos jovens ${ }^{6,7}$. Como o narguilé é considerado uma modalidade de consumo de tabaco, acredita-se que seu uso também possa gerar repercussões na capacidade funcional de seus usuários ${ }^{8}$.

Ainda que seja importante avaliar o efeito da exposição à fumaça de narguilé na função pulmonar, os testes para tal, em repouso podem não demonstrar alterações ${ }^{8}$. Já a avaliação da capacidade de exercício permite analisar respostas metabólicas, respiratórias e cardiovasculares concomitantemente, podendo ser útil na detecção de alterações que emergem apenas em condições dinâmicas ${ }^{9}$.

Recentemente uma revisão sistemática demonstrou que homens cronicamente expostos ao narguilé apresentam alterações na resposta cardiovascular durante o exercício, assim como na capacidade máxima e submáxima de exercício ${ }^{10}$. Apesar disso, a referida revisão não incluiu estudos avaliaram mulheres. Diante deste cenário, o objetivo do presente estudo foi realizar uma revisão integrativa da literatura acerca do comportamento da capacidade funcional em usuários de narguilé de ambos os sexos.

\section{MÉTODOS}

O presente estudo trata-se de uma revisão integrativa da literatura, com buscas conduzidas nas bases de dados Biblioteca Digital Brasileira de Teses e Dissertações (BDTD), Biblioteca Virtual em Saúde (BVS), LILACS, PubMed, SciELO e Scopus no dia 16 de março de 2020. Os descritores usados foram: "smoking water pipes", "tobacco waterpipe", "water pipe smoking", "narghile", "hookah", "shisha", "narguile", "exercise", "aerobic capacity", "exercise tolerance", "exercise test", "walk test". Os operadores booleanos "AND", "ALL" e "OR" foram empregados em conjunto com os descritores mencionados para o rastreamento das publicações. Não foram impostas restrições quanto ao idioma e data de publicação. As estratégias de busca correspondentes a cada base de dados estão descritas na Tabela 1.

Foram incluídos na presente revisão estudos observacionais que: (a) investigaram o efeito do uso agudo e do uso habitual de narguilé sobre a capacidade funcional de indivíduos adultos; (b) utilizaram para avaliação da capacidade funcional um teste padronizado; (c) apresentaram os desfechos dos testes em valores absolutos e relativos. Foram excluídos do presente estudo revisões de literatura, estudos de caso, resumos expandidos, cartas e editoriais.

Dois investigadores analisaram os resultados da pesquisa de modo independente com o intuito de identificar os estudos potencialmente elegíveis. A seleção foi realizada primeiramente pela leitura de todos os títulos, seguida pela leitura dos resumos dos artigos e finalmente

Tabela 1. Estratégias de busca empregadas nas bases de dados.

\begin{tabular}{|c|c|}
\hline Bases de dados & Estratégias de busca \\
\hline Biblioteca Digital Brasileira de Teses e Dissertações (BDTD) & "narguile" \\
\hline Biblioteca Virtual em Saúde (BVS) & $\begin{array}{c}\text { (narghile or hookah or shisha) AND (exercise or aerobic capacity } \\
\text { or walk test) }\end{array}$ \\
\hline LILACS & “narguile" \\
\hline PubMed & $\begin{array}{l}\text { ((smoking water pipes) OR (tobacco, waterpipe) OR (water pipe } \\
\text { smoking)) AND ((exercise tolerance) OR (exercise test) OR (walk } \\
\text { test)) }\end{array}$ \\
\hline SCIELO & "narguile" \\
\hline Scopus & $\begin{array}{l}\text { (ALL(“water pipe smoking") OR ALL("water pipe") OR ALL(hookah) } \\
\text { OR ALL(narghile) OR ALL(shisha)) AND (ALL("exercise tolerance") } \\
\text { OR ALL(“exercise test") OR ALL(“walk test") OR ALL("shuttle walk } \\
\text { test") OR ALL("six minute walk test") OR ALL ("cardiopulmonary } \\
\text { test")) }\end{array}$ \\
\hline
\end{tabular}


com a leitura do artigo na íntegra. Qualquer desacordo entre os revisores foi resolvido por consenso.

Os pesquisadores realizaram a extração dos dados dos estudos incluídos na revisão utilizando um formulário padronizado. As informações coletadas foram: tipo de estudo, número de participantes, sexo, idade, tempo de utilização de narguilé, teste utilizado para avaliação da capacidade funcional, desfechos dos testes em valores absolutos e percentuais, e eventuais variáveis cardiorrespiratórias avaliadas durante os testes.

\section{RESULTADOS}

Ao realizar a busca nas bases de dados foram identificadas 225 publicações e destas, apenas quatro preencheram os critérios de inclusão estabelecidos por esta revisão (Figura 1). Dentre os estudos incluídos, dois avaliaram a capacidade de exercício por meio de teste de esforço cardiopulmonar (CPET) e dois utilizaram o teste de caminhada de 6 minutos (TC6) $)^{8,11-13}$. No total, estes estudos incluíram 171 usuários de narguilé, de ambos os sexos, com faixa etária que oscilou de 18 a 60 anos de idade.

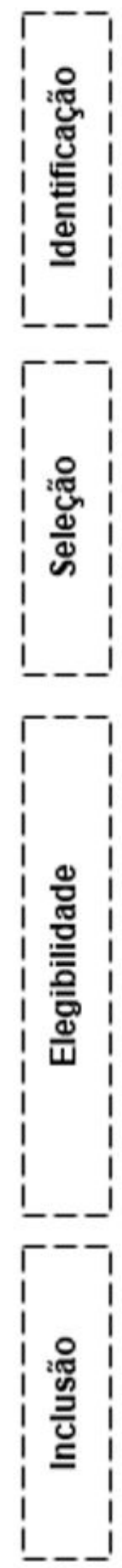

\section{Relatos identificados no banco de dados de buscas $(n=225)$}

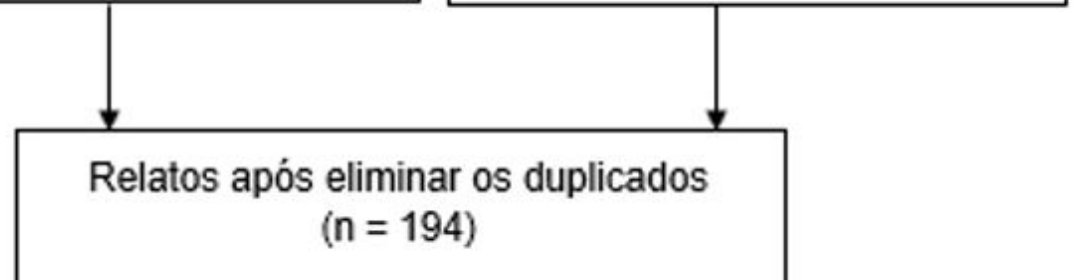

\section{Relatos identificados em outras fontes} $(n=0)$

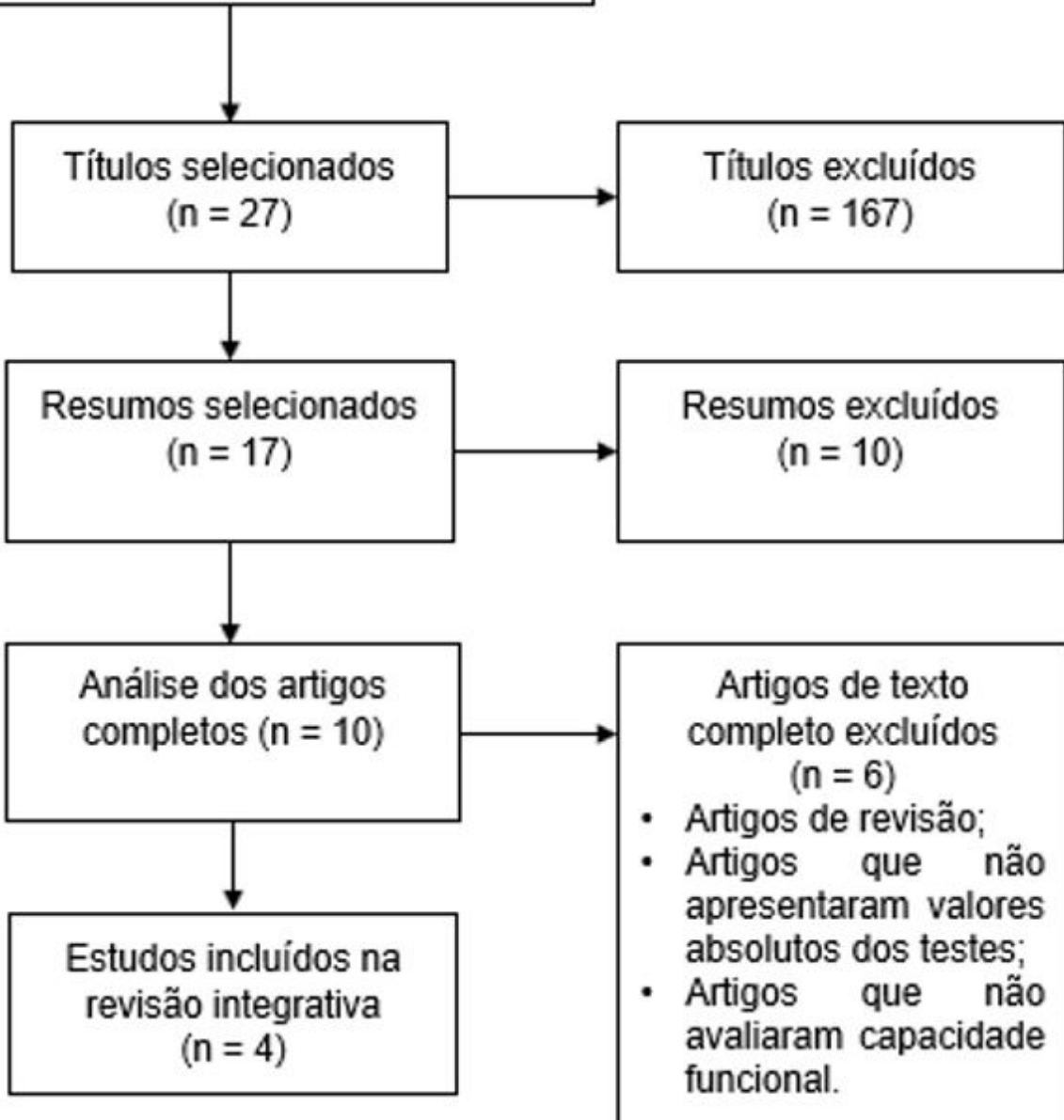

Figura 1. Fluxograma de seleção dos estudos. 
Dois estudos se propuseram a avaliar o efeito habitual do uso de narguilé ${ }^{11,13}$ enquanto outros dois analisaram o efeito agudo do uso de narguilé em indivíduos que rotineiramente já faziam uso dessa modalidade de tabaco (avaliações realizadas imediatamente após uma sessão de 30-45 minutos de narguilé) ${ }^{8,12}$. Demais características dos estudos podem ser observadas na Tabela 2.

Quanto à capacidade funcional, os resultados dos estudos demonstram que o uso habitual de narguilé gera uma redução da capacidade funcional, refletida pela redução do tempo total de exercício ou por menor distância percorrida em seis minutos ${ }^{11,13}$. Além disso, tanto na análise dos efeitos agudos do uso quanto do uso habitual, observou-se que fumantes de narguilé atingem menores valores de $\mathrm{VO}_{2}$ e de frequência cardíaca durante o exercício ${ }^{8,11,13}$. Os estudos também evidenciaram um aumento da dispneia durante a realização dos testes de capacidade funcional8,11,13, entretanto não foram identificadas diferenças estatisticamente significantes no que tange à saturação de oxigênio $0^{8,13}$.

Além disso, na análise dos efeitos agudos do uso de narguilé, observou-se que reduções na distância percorrida no teste de caminhada de seis minutos estiveram associadas à resposta vascular exacerbada durante o exercício. Os resultados de modo detalhado estão dispostos na Tabela 3.

\section{DISCUSSÃO}

O presente estudo buscou fazer um levantamento sobre o comportamento da capacidade funcional em usuários de narguilé. Após análise dos estudos incluídos nesta revisão verificou-se que usuários de narguilé podem apresentar redução significativa da capacidade

Tabela 2. Características dos estudos selecionados publicados entre 2013 e 2017 abordando testes de capacidade aeróbica.

\begin{tabular}{|c|c|c|c|c|c|c|c|c|}
\hline Autor & Ano & Tipo de estudo & Objetivo & $\begin{array}{c}\text { Tamanho da } \\
\text { amostra }\end{array}$ & Sexo & $\begin{array}{l}\text { Idade } \\
\text { (média) }\end{array}$ & $\begin{array}{l}\text { Tempo de uso } \\
\text { de narguilé } \\
\text { (média) }\end{array}$ & Teste utilizado \\
\hline Hawari et al. ${ }^{8}$ & 2013 & Estudo piloto & $\begin{array}{l}\text { Explorar o } \\
\text { possível efeito } \\
\text { da exposição } \\
\text { a fumaça de } \\
\text { narguilé na } \\
\text { capacidade } \\
\text { de exercício } \\
\text { e confirmar o } \\
\text { efeito dessa } \\
\text { exposição } \\
\text { nas medidas } \\
\text { da função } \\
\text { pulmonar }\end{array}$ & 24 fumantes & Masculino & $\begin{array}{c}18 \text { a } 25 \text { anos } \\
(20,4 \text { anos })\end{array}$ & 3,7 anos & $\begin{array}{l}\text { Teste de esforço } \\
\text { cardiopulmonar } \\
\text { limitado por } \\
\text { tempo }\end{array}$ \\
\hline Ben Saad et al. ${ }^{13}$ & 2014 & $\begin{array}{c}\text { Estudo } \\
\text { transversal }\end{array}$ & $\begin{array}{c}\text { Avaliar e } \\
\text { comparar a } \\
\text { capacidade } \\
\text { aeróbica } \\
\text { submáxima de } \\
\text { um grupo de } \\
\text { fumantes de } \\
\text { narguilé e um } \\
\text { grupo controle } \\
\text { de indivíduos } \\
\text { saudáveis, de } \\
\text { mesma idade }\end{array}$ & $\begin{array}{c}78 \\
\text { Fumantes: } 25 \\
\text { Não fumantes: } \\
53\end{array}$ & Masculino & $\begin{array}{l}40 \text { a } 60 \text { anos } \\
\text { (fumantes: } \\
47 \text { anos; não } \\
\text { fumantes: } 49 \\
\text { anos). }\end{array}$ & 5 anos & $\begin{array}{c}\text { Teste de } \\
\text { caminhada de } 6 \\
\text { minutos }\end{array}$ \\
\hline Alomari et al. ${ }^{12}$ & 2015 & Não relatado & $\begin{array}{c}\text { Avaliar as } \\
\text { mudanças } \\
\text { agudas } \\
\text { induzidas pelo } \\
\text { uso do narguilé } \\
\text { na função } \\
\text { vascular em } \\
\text { jovens saudáveis }\end{array}$ & $\begin{array}{l}53 \text { indivíduos } \\
\text { que fumaram } \\
\text { apenas para } \\
\text { realizar a } \\
\text { avaliação }\end{array}$ & $\begin{array}{l}\text { Masculino: } 39 \\
\text { Feminino: } 19\end{array}$ & $\begin{array}{c}18 \text { a } 35 \text { anos } \\
(22,7 \text { anos } \pm 4,8)\end{array}$ & Não relatado & $\begin{array}{c}\text { Teste de } \\
\text { caminhada de } 6 \\
\text { minutos }\end{array}$ \\
\hline Hawari et al. ${ }^{11}$ & 2017 & $\begin{array}{l}\text { Estudo piloto } \\
\text { exploratório }\end{array}$ & $\begin{array}{l}\text { Comparar } \\
\text { os fumantes } \\
\text { de narguilé } \\
\text { com os não } \\
\text { fumantes em } \\
\text { três áreas: carga } \\
\text { de sintomas } \\
\text { respiratórios, } \\
\text { função } \\
\text { pulmonar e } \\
\text { capacidade } \\
\text { de exercício } \\
\text { cardiopulmonar }\end{array}$ & $\begin{array}{c}\text { Total: } 138 \\
\text { Fumantes: } 69 \\
\text { Não fumantes: } \\
69\end{array}$ & Masculino & $\begin{array}{l}18 \text { a } 26 \text { anos } \\
\text { (fumantes: } \\
\text { 22,1 anos; não } \\
\text { fumantes: } 21,4 \\
\text { anos) }\end{array}$ & 4,9 anos & $\begin{array}{l}\text { Teste de esforço } \\
\text { cardiopulmonar } \\
\text { limitado por } \\
\text { sintomas }\end{array}$ \\
\hline
\end{tabular}


Tabela 3. Resultados dos estudos selecionados publicados entre 2013 e 2017 abordando teste de capacidade aeróbica.

\begin{tabular}{|c|c|c|c|c|c|c|c|}
\hline Autor & Ano & $\begin{array}{c}\text { Distância } \\
\text { percorrida }\end{array}$ & $\begin{array}{l}\text { Saturação de } \\
\text { oxigênio }\end{array}$ & $\begin{array}{c}\text { Frequência } \\
\text { cardíaca }\end{array}$ & Dispneia & $\begin{array}{l}\text { Tempo de } \\
\text { exercício (em } \\
\text { minutos) }\end{array}$ & $\begin{array}{l}\text { Consumo de } \\
\text { oxigênio }\left(\mathrm{VO}_{2}\right)\end{array}$ \\
\hline Hawari et al. ${ }^{8}$ & 2013 & Não foi avaliada & $\begin{array}{c}\text { 96,4\% no período } \\
\text { pré exposição } \\
\text { versus } 96,1 \% \\
\text { no período pós } \\
\text { exposição ( } \mathrm{p}> \\
0,05)\end{array}$ & $\begin{array}{c}\text { Basal: 82,9 bpm } \\
\text { no período pré } \\
\text { exposição versus } \\
85,3 \text { bpm no } \\
\text { período pós } \\
\text { exposição Máxima } \\
\text { atingida: 179,3 } \\
\text { bpm no período } \\
\text { pré exposição } \\
\text { versus 178,5 bpm } \\
\text { no período pós } \\
\text { exposição ( } p> \\
0,05)\end{array}$ & $\begin{array}{c}\text { Avaliada pela } \\
\text { Escala de Borg no } \\
\text { pico do exercício: } \\
\text { 4,4 no período pré } \\
\text { exposição versus } \\
5,2 \text { no período } \\
\text { pós exposição ( } p \\
<0,05)\end{array}$ & $\begin{array}{c}\text { 9,4 min de } \\
\text { exercício concluído } \\
\text { no período } \\
\text { pré exposição } \\
\text { versus } 9,2 \text { min } \\
\text { de exercício no } \\
\text { período pós } \\
\text { exposição }\end{array}$ & $\begin{array}{c}\text { 1,86 L/min no } \\
\text { período pré } \\
\text { exposição versus } \\
\text { 1,7 L/min período } \\
\text { pós exposição ( } p \\
<0,05)\end{array}$ \\
\hline Ben Saad et al..$^{13}$ & 2014 & $\begin{array}{c}654 \text { metros ( } 87 \% \\
\text { do previsto) nos } \\
\text { fumantes versus } \\
756 \text { metros ( } 98 \% \\
\text { do previsto) nos } \\
\text { não fumantes ( } p= \\
0,001 \text { ) }\end{array}$ & $\begin{array}{l}\text { Basal: } 97 \% \text { nos } \\
\text { fumantes versus } \\
98 \% \text { nos não } \\
\text { fumantes ( } p= \\
\text { 0,04) Final: } 97 \% \\
\text { nos fumantes } \\
\text { versus } 97 \% \text { nos } \\
\text { não fumantes ( } p \\
=0,48 \text { ) }\end{array}$ & $\begin{array}{c}\text { Basal: } 82 \text { bpm nos } \\
\text { fumantes versus } \\
74 \text { bpm nos não } \\
\text { fumantes Final: } \\
120 \text { bpm nos } \\
\text { fumantes versus } \\
154 \text { bpm nos não } \\
\text { fumantes ( } p= \\
0,001)\end{array}$ & $\begin{array}{l}\text { Avaliada por escala } \\
\text { visual analógica } \\
\text { de } 0 \text { a } 10 \text { : Basal: } \\
0,1 \text { nos fumantes } \\
\text { versus } 0,0 \text { nos } \\
\text { não fumantes } \\
\text { ( } p=0,008 \text { ) Final: } \\
2,3 \text { nos fumantes } \\
\text { versus } 1,0 \text { nos não } \\
\text { fumantes ( } p= \\
0,001)\end{array}$ & Não se aplica & Não foi avaliado \\
\hline Alomari et al. ${ }^{12}$ & 2015 & $\begin{array}{c}614,8 \pm 129,9 \\
\text { metros }\end{array}$ & Não foi avaliada & Não foi avaliada & Não foi avaliada & Não se aplica & Não foi avaliado \\
\hline Hawari et al. ${ }^{11}$ & 2017 & Não foi avaliada & Não foi avaliada & $\begin{array}{c}87 \% \text { do previsto } \\
\text { em fumantes de } \\
\text { narguilé versus } \\
93,1 \% \text { em não } \\
\text { fumantes ( } p= \\
0,001 \text { ) }\end{array}$ & $\begin{array}{c}\text { Avaliada pela } \\
\text { Escala de Borg: } \\
\text { 5,4 em fumantes } \\
\text { versus } 3,9 \text { em não } \\
\text { fumantes ( } p= \\
0,001)\end{array}$ & $\begin{array}{c}8,5 \text { min nos } \\
\text { fumantes versus } \\
8,9 \text { min nos não } \\
\text { fumantes }(p=0,03)\end{array}$ & $\begin{array}{c}57,5 \% \text { do previsto } \\
\text { nos fumantes } \\
\text { versus } 61,2 \% \\
\text { do previsto não } \\
\text { fumantes }(p=0,02)\end{array}$ \\
\hline
\end{tabular}

min: minutos; bpm: batimentos por minuto; L/min: litros por minuto; p: p-valor.

de exercício e do consumo de oxigênio, atingindo maiores valores de frequência cardíaca, com percepção de dispneia mais elevada.

Os achados da presente revisão podem ser explicados por alguns mecanismos associados à presença de monóxido de carbono (CO) no sangue e os efeitos da nicotina. $A$ exposição à fumaça de narguilé resulta no aumento dos níveis de CO, gás que modula a curva de dissociação da oxi-hemoglobina, alterando a forma e posição das curvas de saturação. A afinidade do CO pela hemoglobina é 240 vezes maior do que do oxigênio, então, quantidades pequenas de CO reduzem de modo considerável a capacidade da hemoglobina de carrear oxigênio. Esse fato gera uma redução da pressão parcial de oxigênio no sangue, fato que gera prejuízo na distribuição deste gás para os tecidos e consequentemente, a captação do mesmo pelo músculo em exercício ${ }^{14}$. Ao receber menos oxigênio, os músculos requeridos durante uma atividade produzem menos ATP, limitando a capacidade de realização dos exercícios.

Além disso, a nicotina, substância presente no narguilé, é um alcaloide que age através da barreira cerebral, se ligando a receptores nicotínicos no sistema nervoso central. Por meio destes receptores, a nicotina permite a liberação da adrenalina, estimulando o aumento da contração cardíaca e dos vasos sanguíneos, resultando em elevação da frequência cardíaca e pressão arterial. Além disso, a nicotina ainda produz a constrição das vias aéreas, causando falta de ar e taquipneia, através da estimulação dos gânglios simpáticos nos brônquios ${ }^{15,16}$.

Em comparação a um único cigarro, uma sessão de narguilé está associada a níveis similares de nicotina no plasma e níveis três vezes maiores de carboxihemoglobina. Nos primeiros cinco minutos de uso de narguilé, observa-se uma produção quatro vezes maior de carboxihemoglobina do que quando se fuma apenas um cigarro. Apesar das concentrações máximas de nicotina sejam semelhantes entre cigarro e narguilé, deve-se levar em consideração que uma sessão de narguilé dura cerca de 45 minutos, muito mais tempo de que fumar um único cigarro ${ }^{17}$. Por este motivo postula-se que o uso de narguilé possa causar impactos negativos mais acentuados na capacidade funcional do que o cigarro. Essa hipótese também é fortalecida pelos dados apresentados por Hawari e colaboradores (2017), os quais sugerem que uma média de cinco anos de uso habitual de narguilé está associado com a capacidade de exercício reduzida, o que é um tempo relativamente curto se comparado ao uso do cigarro"1.

Além do uso de narguilé produzir uma considerável exposição à nicotina e monóxido de carbono, ele também 
envolve inalação de muitos litros de fumaça (cerca de 40 vezes mais que um cigarro), que possui vários outros tóxicos, como hidrocarbonetos aromáticos policíclicos, aldeídos voláteis e metais pesados, que são causadores de doenças pulmonares ${ }^{17,18}$.

Já tem sido demonstrado que usuários de narguilé apresentam um comprometimento da função pulmonar, evidenciado pela redução da capacidade vital forçada, do volume expiratório forçado no primeiro segundo e da relação entre estas duas variáveis ${ }^{19}$. Com base nesses achados alguns autores argumentam que tanto o narguilé quanto o cigarro possuem um impacto igualmente negativo sobre a função pulmonar, e que o uso crônico dessas substâncias deve ser considerado como um fator de risco potencial para o desenvolvimento de doenças pulmonares obstrutivas ${ }^{19}$. Como as alterações da função pulmonar predispõem à ocorrência do declínio na capacidade funcional, é possível que os resultados encontrados na presente revisão também possam ser justificados com base nessa pressuposição.

O presente estudo possui algumas limitações. Apesar da busca por informações ter sido realizada de modo sistemático em diversas bases de dados, apenas quatro estudos se enquadraram nos critérios de inclusão estabelecidos. Além disso, os delineamentos das pesquisas foram bastante heterogêneos o que impediu a utilização de um instrumento para avaliar a qualidade das investigações incluídas nesta revisão. Apesar disso, ao que se sabe, este é um dos primeiros estudos que se propôs a compilar evidências sobre as consequências do narguilé sobre a capacidade funcional em indivíduos de ambos os sexos. Os dados levantados por esta revisão reforçam a necessidade de ações de conscientização sobre os malefícios causados por esta modalidade de tabagismo.

\section{CONCLUSÃO}

Os achados desta revisão apontam que indivíduos que fumam narguilé podem apresentar redução significativa da capacidade de exercício e do consumo de oxigênio. Associada a esta redução, observa-se também elevações da frequência cardíaca e da sensação de dispneia. Estas evidências reforçam o impacto negativo que o uso de narguilé causa na saúde dos indivíduos.

\section{FONTE DE FINANCIAMENTO}

Nada a declarar.

\section{CONFLITO DE INTERESSES}

Nada a declarar.

\section{REFERÊNCIAS}

1. ANVISA: Agência Nacional de Vigilância Sanitária. Danos à saúde Narguilé [Internet]. [citado em 2020 junho 03]. Disponível em: http://portal.anvisa.gov.br/tabaco/narguile 2. INCA: Instituto Nacional de Câncer José Alencar Gomes da Silva. Uso de narguilé: efeitos sobre a saúde, necessidades de pesquisa e ações recomendadas para legisladores. Rio de Janeiro: INCA; 2017.

3. Akl EA, Gaddam S, Gunukula SK, Honeine R, Jaoude PA, Irani J. The effects of waterpipe tobacco smoking on health outcomes: a systematic review. Int J Epidemiol. 2010;39(3):834-57. http://dx.doi.org/10.1093/ije/dyq002. PMid:20207606.

4. Jaber R, Madhivanan P, Veledar E, Khader Y, Mzayek F, Maziak W. Waterpipe a gateway to cigarette smoking initiation among adolescents in Irbid, Jordan: a longitudinal study. Int J Tuberc Lung Dis. 2015;19(4):481-7. http://dx.doi. org/10.5588/ijtld.14.0869. PMid:25860006.

5. Maziak W. Commentary: the waterpipe-a global epidemic or a passing fad. Int J Epidemiol. 2010;39(3):857-9. http:// dx.doi.org/10.1093/ije/dyq054. PMid:20348109.

6. Lee C-L, Chang WD. The effects of cigarette smoking on aerobic and anaerobic capacity and heart rate variability among female university students. Int J Womens Health. 2013;5:667-79. http://dx.doi.org/10.2147/IJWH.S49220. PMid:24204174.

7. Sumartiningsih S, Lin H-F, Lin J-C. Cigarette smoking blunts exercise-induced heart rate response among young adult male smokers. Int J Environ Res Public Health. 2019;16(6):1032. http://dx.doi.org/10.3390/ijerph16061032. PMid:30901920.

8. Hawari Fl, Obeidat NA, Ayub H, Ghonimat I, Eissenberg T, Dawahrah S, et al. The acute effects of waterpipe smoking on lung function and exercise capacity in a pilot study of healthy participants. Inhal Toxicol. 2013;25(9):492-7. http://dx.doi. org/10.3109/08958378.2013.806613. PMid:23905967.

9. Neder JA, Nery LE. Teste de exercício cardiopulmonar. J Pneumol. 2002;28(3):166-206.

10. Chaieb F, Ben Saad H. The chronic effects of narghile use on males' cardiovascular response during exercise: a systematic review. Am J Men's Health. 2021;15(2):1-18. http://dx.doi. org/10.1177/1557988321997706. PMid:33729068.

11. Hawari Fl, Obeidat NA, Ghonimat IM, Ayub HS, Dawahreh SS. The effect of habitual waterpipe tobacco smoking on pulmonary function and exercise capacity in young healthy males: a pilot study. Respir Med. 2017;122:71-5. http:// dx.doi.org/10.1016/j.rmed.2016.11.024. PMid:27993294.

12. Alomari MA, Khabour OF, Alzoubi KH, Shqair DM, Stoner L. Acute vascular effects of waterpipe smoking: importance of physical activity and fitness status. Atherosclerosis. 2015;240(2):472-6. http://dx.doi.org/10.1016/j. atherosclerosis.2015.02.047. PMid:25909821.

13. Ben Saad H, Babba M, Boukamcha R, Ghannouchi I, Latiri I, Mezghenni S, et al. Investigation of exclusive narghile smokers: deficiency and incapacity measured by spirometry and 6-minute walk test. Respir Care. 2014;59(11):1696-709. http://dx.doi.org/10.4187/respcare.03058. PMid:25006270.

14. Powell FL. Oxygen and carbon dioxide transport in the blood. In: Johnson LR, editor. Essential medical physiology. Burlington: Elsevier Academic Press; 2003. p. 290-98.

15. Shaikh RB, Vijayaraghavan N, Sulaiman AS, Kazi S, Shafi MSM. The acute effects of waterpipe smoking on the cardiovascular and respiratory systems. J Prev Med Hyg. 2008;49(3):101-7. PMid:19278135. 
16. Hakim F, Hellou E, Goldbart A, Katz R, Bentur Y, Bentur $L$. The acute effects of water-pipe smoking on the cardiorespiratory system. Chest. 2011;139(4):77581. http://dx.doi.org/10.1378/chest.10-1833. PMid:21030492.

17. Eissenberg T, Shihadeh A. Waterpipe tobacco and cigarette smoking: direct comparison of toxicant exposure. Am J Prev Med. 2009;37(6):518-23. http://dx.doi.org/10.1016/j. amepre.2009.07.014. PMid:19944918.
18. Cobb CO, Shihadeh A, Weaver MF, Eissenberg T. Waterpipe tobacco smoking and cigarette smoking: a direct comparison of toxicant exposure and subjective effects. Nicotine Tob Res. 2011;13(2):78-87. http://dx.doi.org/10.1093/ntr/ntq212. PMid:21127030.

19. Patel MP, Khangoora VS, Marik PE. A review of the pulmonary and health impacts of hookah use. Ann Am Thorac Soc. 2019;16(10):1215-9. http://dx.doi.org/10.1513/ AnnalsATS.201902-129CME. PMid:31091965. 